\title{
Tracking the Fear Engram: The Lateral Amygdala Is an Essential Locus of Fear Memory Storage
}

\author{
Glenn E. Schafe, ${ }^{1}$ Valérie Doyère, ${ }^{2,3}$ and Joseph E. LeDoux ${ }^{3}$ \\ ${ }^{1}$ Department of Psychology and Interdepartmental Neuroscience Program, Yale University, New Haven, Connecticut 06520, ${ }^{2}$ Laboratoire de Neurobiologic \\ de l'Apprentissage, de la Mémoire, et de la Communication, Unité Mixte de Recherche 8620, Centre National de la Recherche Scientifique-Université \\ Paris-Sud, 91405 Orsay, France, and ${ }^{3}$ Center for Neural Science, New York University, New York, New York 10003
}

\begin{abstract}
Although it is believed that different types of memories are localized in discreet regions of the brain, concrete experimental evidence of the existence of such engrams is often elusive. Despite being one of the best characterized memory systems of the brain, the question of where fear memories are localized in the brain remains a hotly debated issue. Here, we combine site-specific behavioral pharmacology with multisite electrophysiological recording techniques to show that the lateral nucleus of the amygdala, long thought to be critical for the acquisition of fear memories, is also an essential locus of fear memory storage.
\end{abstract}

Key words: fear conditioning; memory consolidation; amygdala; synaptic plasticity; rat; MAP kinase

\section{Introduction}

The fear-learning system of the brain has generated enormous interest, attributable in part to its attractiveness as a neurobiological model of learning and memory and also to its potential clinical significance (Davis, 1992; LeDoux, 2000). Despite years of progress, however, there is still no universal consensus regarding the locus of fear memory storage (Cahill et al., 1999; Fanselow and LeDoux, 1999; Paré, 2002).

Most work on the neural system of fear learning has involved studies of auditory pavlovian fear conditioning (Fanselow and LeDoux, 1999; LeDoux, 2000), a learning paradigm in which an emotionally neutral auditory conditioned stimulus (CS) comes to elicit fear after it is paired with an aversive unconditioned stimulus (US). This research has led to the view that the lateral nucleus of the amygdala (LA) is a key site of plasticity underlying fear learning (LeDoux, 2000; Blair et al., 2001; Maren and Quirk, 2004). Several independent lines of evidence have supported this view. These have included electrophysiological recording studies showing that CS and US pathways converge onto single cells in the LA and that paired presentations of the CS and US enhance the response to the CS in LA cells (Romanski et al., 1993; Quirk et al., 1995; Rogan et al., 1997), lesion studies showing that damage to or inactivation of the LA prevents fear conditioning (LeDoux, 2000), and pharmacological studies showing that blockade of intracellular signaling cascades in the LA that are essential for either short- or long-term synaptic plasticity impairs short-term

Received July 1, 2005; revised September 17, 2005; accepted September 18, 2005.

This work was supported by National Institute of Mental Health (NIMH) Grants MH46516, MH00956, MH39774, MH11902, and MH570161 (J.E.L.), Centre National de la Recherche Scientifique/NSF Cooperative Grant 17089 (V.D. and J.E.L.), NIMH Grant MH62519 (G.E.S.), and by a grant from the W. M. Keck Foundation to New York University. We thank Jeffrey Erlich, Elizabeth Bauer, Tad Blair, and Jean-Marc Edeline for helpful discussions about this manuscript and Yu Zhou for assistance with making electrodes.

Correspondence should be addressed to Dr. Glenn E. Schafe, Department of Psychology, Yale University, 2 Hillhouse Avenue, Box 208205, New Haven, CT 06520. E-mail: glenn.schafe@yale.edu.

DOI:10.1523/JNEUROSCI.3307-05.2005

Copyright $\odot 2005$ Society for Neuroscience $\quad$ 0270-6474/05/2510010-06\$15.00/0 memory (STM) and long-term memory (LTM) of fear conditioning, respectively (Rodrigues et al., 2004).

An alternative view argues that the LA is not a site of fear memory storage and offers alternative interpretations of each of the aforementioned findings (Cahill et al., 1999). For example, it has been pointed out that the LA is not unique but rather one of many regions of the wider fear network to exhibit neurophysiological changes during and after fear conditioning. Indeed, auditory fear conditioning induces alterations in the activity of neurons not only in the LA but also in the auditory cortex (Bakin and Weinberger, 1990; Edeline and Weinberger 1993) and the auditory thalamus (Gabriel et al., 1975; Lennartz and Weinberger, 1992). These findings suggest the possibility that the neurophysiological changes observed in the LA during and after fear conditioning might merely be a reflection of plastic changes that have occurred in these upstream regions rather than representing a neural signature of memory storage in the LA itself (Cahill et al., 1999). Furthermore, rather than indicating that the LA is a site of storage of fear memories, it has been suggested that memory deficits observed after lesion and pharmacological manipulations may instead indicate that the LA is essential for triggering or modulating the strength of plasticity and memory storage in other regions of the wider fear network (Cahill et al., 1999).

It has become clear that lesion, electrophysiological, or pharmacological methods alone are not sufficient to identify the locus of fear memory storage. In the present study, we have therefore taken a new approach to tracking the fear engram by combining a site-specific pharmacological manipulation of the LA that targets memory consolidation processes with simultaneous multisite electrophysiological recordings in different regions of the fear memory system.

\section{Materials and Methods}

Subjects. Adult male Sprague Dawley rats (Hilltop Laboratories, Philadelphia, PA) were housed individually with ad libitum food and water on a 
$12 \mathrm{~h}$ light/dark cycle. All procedures were approved by the New York University Animal Care and Use Committee.

Surgery. Rats were anesthetized with Nembutal (45 mg/kg, i.p.) and implanted bilaterally with cannulas (26 gauge; Plastics One, Roanoke, VA) aimed at the LA [anteroposterior (AP), -3.2; mediolateral (ML), \pm 5.2 ; dorsoventral (DV), -8.0 ] and a bipolar stimulation electrode (250 $\mu \mathrm{m}$; Rhodes Medical, Summerland, CA) aimed at the left auditory thalamus [medial geniculate nucleus/posterior intralaminar nucleus (MGm/ PIN); AP, -5.4 ; ML, $\pm 3.0 ; \mathrm{DV},-6.6]$. Attached to the left cannula was an insulated stainless steel recording wire $(1 \mathrm{M} \Omega)$ that extended $\sim 0.5$ $\mathrm{mm}$ from the base of the infusion cannula. The final depth was determined using stimulation of the MGm/PIN as a guide (Doyère et al., 2003). Two cortical silver balls, placed contralaterally, served as a reference and ground, and dental cement was used to anchor the electrodes and connecting device to the skull. Rats were administered buprenorphine- $\mathrm{HCl}(0.02 \mathrm{mg} / \mathrm{kg})$ for analgesia and given at least 1 week to recover.

Electrophysiological and behavioral procedures. Rats were habituated to the conditioning chamber and to cable connection and given $2 \mathrm{~d}$ of pre-exposure to three $20 \mathrm{~s}$ CSs $(1 \mathrm{kHz}, 50 \mathrm{~ms}$ tone pips, $80 \mathrm{~dB}$, at $1 \mathrm{~Hz})$, with a variable intertrial interval (ITI) of $160 \mathrm{~s}$, on average. On the fourth day, rats were infused bilaterally with either 1,4-diamino-2,3-dicyano1,4-bis(2-aminophenylthio)-butadiene (U0126) ( $1 \mu \mathrm{g} ; 0.5 \mu \mathrm{l} ; n=8)$ or an equivalent volume of $50 \%$ DMSO vehicle $(n=5)$. This dose of U0126 was chosen based on findings showing that it reliably impairs fear memory consolidation and the activation of extracellular signal-regulated kinase (ERK)/mitogen-activated protein kinase (MAPK) in the LA (Schafe et al., 2000).

Thirty minutes after infusion, rats received five CS-US pairings (ITI, $\sim 130 \mathrm{~s}$ ), each CS terminating with a $0.5 \mathrm{~s}, 1 \mathrm{~mA}$ footshock (US). Retention of fear memory was then tested in a distinct chamber both at $3 \mathrm{~h}$ (STM; three CSs) and at $24 \mathrm{~h}$ (LTM; $10 \mathrm{CSs}$ ). During pre-exposure and retention tests, auditory-evoked field potentials (AEFPs) were recorded from both the LA and MGm/PIN (through the tip of the stimulation electrode). For technical reasons, we were able to record simultaneously from both the LA and MGm/PIN in a subset of rats (vehicle, $n=3$; U0126, $n=6$ ).

Data analysis. The rats' freezing behavior was recorded onto videotape during all sessions for off-line scoring. AEFPs were recorded and analyzed using DataWave and averaged across all tone-pip presentations within a trial and collapsed across trials. The amplitude of the shortlatency negative component was measured for each potential $(\sim 12-15$ $\mathrm{ms}$ for the LA; $\sim 7-9 \mathrm{~ms}$ for the MGm/PIN). We assessed retention of behavioral and electrophysiological changes by expressing the change during the last three trials of the LTM test as a percentage of that during the three trials of the STM test. This measure proved convenient for directly correlating the maintenance of changes at both behavioral and neurophysiological levels within the same animal.

Histology. At the end of the experiment, the location of the electrodes was marked by passing a small amount of anodal current $(4 \mu \mathrm{A} ; 5 \mathrm{~s})$ through the electrode tips. Rats were then perfused with $2 \%$ potassium ferrocyanide, and brains were sectioned and stained with Nissl for analysis of electrode placement.

\section{Results}

\section{A strategy for tracking the fear engram}

Fear conditioning leads to neurophysiological changes in LA neurons, a finding that may reflect plasticity either locally at LA synapses (Quirk et al., 1996; Blair et al., 2001) or at structures that are upstream of the LA, such as the auditory thalamus or cortex (Cahill et al., 1999). Lesions of auditory cortical regions have no effect on fear conditioning to simple auditory cues (Romanski and LeDoux, 1992), and the latency of plastic changes in the auditory cortex is too long to account for changes in the LA (Quirk et al., 1996). However, it remains plausible that the auditory thalamic areas that project to the LA, including the MGm/ PIN, are principal sites of fear memory storage and that the neu-
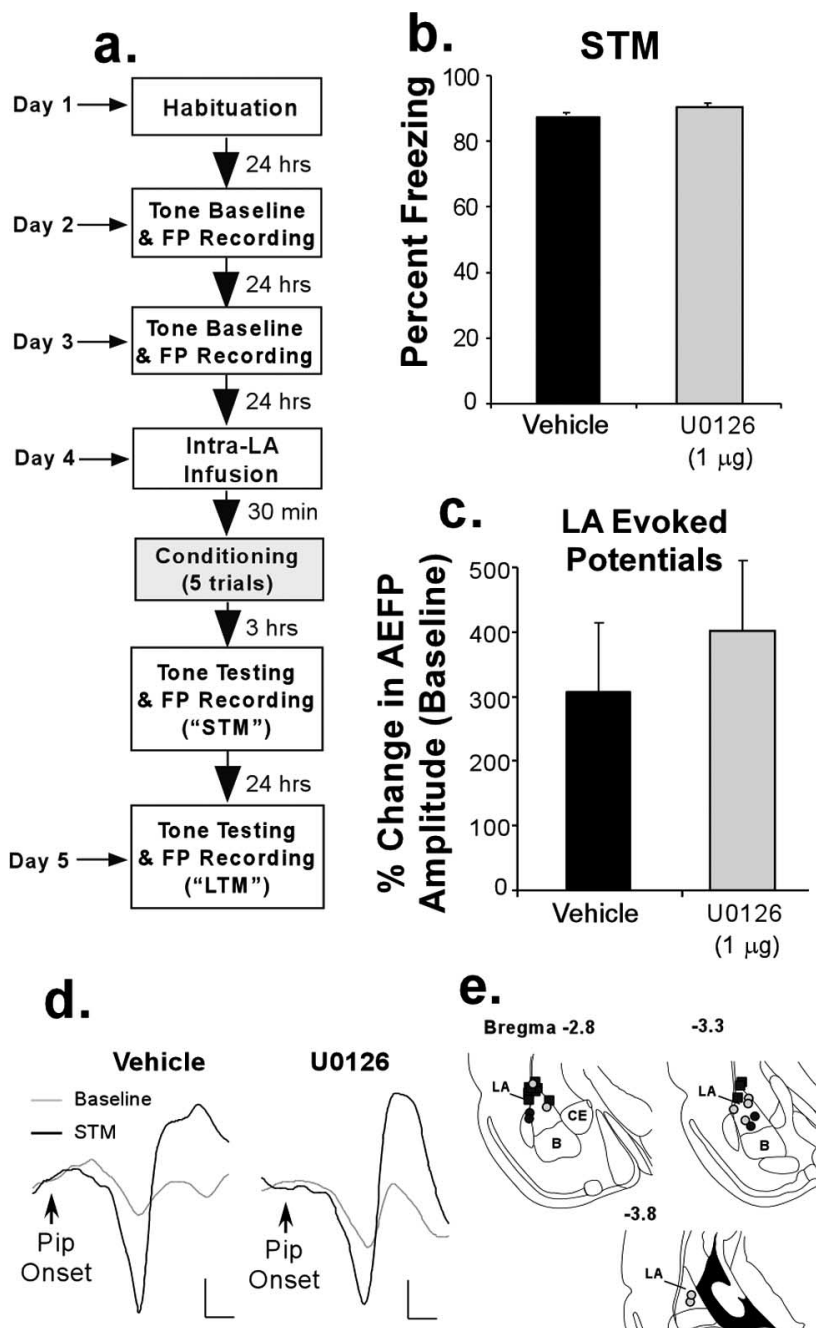

e.

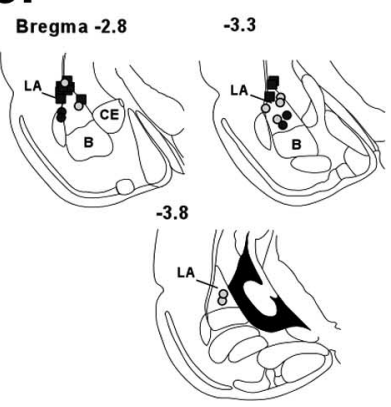

Figure 1. Intra-LA infusion of a MAPK inhibitor has no effect on STM formation of fear conditioning and conditioning-induced synaptic plasticity in the LA. $\boldsymbol{a}$, Schematic of the experimental protocol. $\boldsymbol{b}$, Mean ( \pm SEM) percentage of freezing expressed during the STM test in rats treated with $50 \%$ DMSO vehicle ( $n=5$; black bar) or $1 \mu$ g of U0126 ( $n=8$; gray bar). c, Mean ( \pm SEM) percentage of change in AEFP amplitude in the LA for each group during the STM test, relative to baseline. $\boldsymbol{d}$, Representative AEFPs in the LA for each group (vehicle, U0126) during baseline and during the STM test. Calibration: $20 \mu \mathrm{V}, 5 \mathrm{~ms}$. $\boldsymbol{e}$, Electrode placements in rats treated with vehicle (black circles) or U0126 (gray circles). Cannula tip placements are shown as black squares. B, Basal nucleus of the amygdala; $C E$, central nucleus of the amygdala.

rophysiological changes observed in the LA simply reflect a "read-out" from these regions.

To address this question, we have combined simultaneous electrophysiological recordings from both the LA and MGm/PIN in freely behaving rats with an intra-LA infusion of an inhibitor of ERK/MAPK, a pharmacological treatment that blocks intracellular signaling cascades necessary for synaptic plasticity and impairs long-term consolidation of auditory fear conditioning (Schafe et al., 2000). Rats were given an intra-LA infusion of U0126, an inhibitor of MEK (an upstream regulator of ERK/ MAPK), or an equivalent volume of 50\% DMSO vehicle. They were then conditioned and tested for both STM (assessed $3 \mathrm{~h}$ after training) and LTM (assessed $\sim 24 \mathrm{~h}$ after training) while recordings were made from both the LA and MGm/PIN (Fig. 1a).

This simple, unique combination of techniques allows for simultaneous measurement of learning-induced changes at both 
ends of the monosynaptic MGm/PIN-LA pathway over a period of $24 \mathrm{~h}$ after intra-LA infusion of a compound known to impair fear memory formation. Importantly, the MEK inhibitor has no effect on fear acquisition or STM; it only affects the conversion of STM to LTM (Schafe et al., 2000). Plastic changes should therefore be free to develop across the wider fear network during the acquisition process. Inhibition of MEK will, however, disrupt the consolidation process during which short-term plasticity is converted into long-term plasticity for LTM storage while we track the neurophysiological correlates of this process at both thalamic and LA recording sites.

We reasoned that if LA synapses are the critical site of plasticity underlying memory formation and storage of fear conditioning, then local inhibition of ERK/MAPK activation in the LA during training should selectively impair both fear memory consolidation and the consolidation of conditioning-induced synaptic plasticity in the LA, while having no effect on the establishment or long-term retention of thalamic plasticity.

Intra-LA infusion of a MAPK inhibitor impairs consolidation of fear conditioning and conditioning-induced synaptic plasticity in the LA

Rats given an intra-LA infusion of U0126 before conditioning exhibited intact STM relative to controls $\left(t_{(11)}=0.46 ; p>0.05\right)$ (Fig. 1b). Furthermore, fear conditioning led to significant enhancement of the activity of LA neurons. This was reflected as an increase in the amplitude of AEFPs in the LA, which was equivalent in both the vehicle and U0126-treated groups (Fig. 1c,d). This enhancement was significant relative to baseline (vehicle: $t_{(4)}$ $=2.86, p<0.05 ; \mathrm{U} 0126: t_{(7)}=7.18, p<0.01$ ), and the groups did not differ $\left(t_{(11)}=0.59 ; p>0.05\right)$. Representative traces from vehicle and U0126-treated rats are shown in Figure 1d, whereas electrode placements are shown in Figure 1e.

In contrast to the STM test, U0126-treated rats exhibited impaired $\operatorname{LTM}\left(t_{(11)}=2.28 ; p<0.05\right)($ Fig. $2 a)$. This difference was also observed when LTM was expressed as a percentage of STM for each rat $\left(t_{(11)}=2.86 ; p<0.05\right)$ (Fig. $\left.2 b\right)$. Neurophysiological responses recorded from the LA followed the same pattern (Fig. $2 c)$. For this analysis, we expressed the amplitude change during the LTM test as a percentage of that during the STM test for each animal. Relative to vehicle controls, this analysis revealed that U0126-treated rats exhibited impaired long-term retention of neurophysiological changes in the LA $\left(t_{(11)}=3.17 ; p<0.05\right)$. To further examine this effect, we performed a regression analysis within the U0126-treated group between freezing scores and percentage of change in AEFP amplitudes (each expressed as a percentage of STM). The analysis revealed a highly significant correlation $\left(r_{(7)}=0.92 ; p<0.01\right)$ (Fig. $2 d$ ), indicating that the more effective U0126 was at impairing the consolidation of fear memory, the more effective it was at impairing the consolidation of conditioning-induced synaptic plasticity in the LA. Representative traces recorded at $24 \mathrm{~h}$ from the LA in vehicle and U0126treated rats are shown in Figure $2 e$.

These findings indicate that local inhibition of ERK/MAPK activation in the LA impairs the consolidation of both fear memory and conditioning-induced changes in the LA, a finding consistent with the idea that the plasticity underlying the consolidation of fear conditioning occurs locally at LA synapses.

Intra-LA infusion of a MAPK inhibitor does not impair conditioning-induced synaptic plasticity in the MGm/PIN The finding that an intra-LA infusion of an inhibitor of ERK/ MAPK impairs long-term synaptic plasticity and fear memory
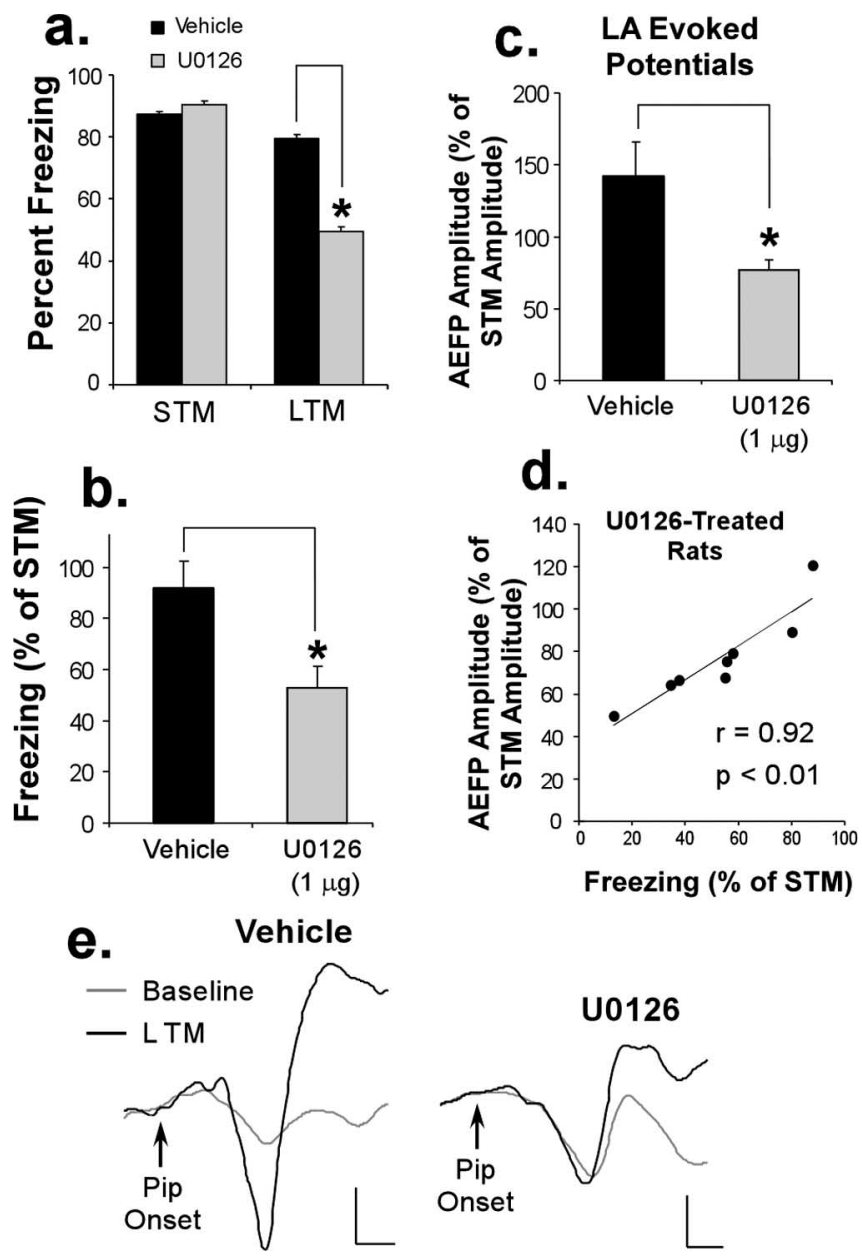

Figure 2. Intra-LA infusion of a MAPK inhibitor impairs LTM of fear conditioning and conditioning-induced synaptic plasticity in the LA. $\boldsymbol{a}$, Mean ( \pm SEM) percentage of freezing expressed during the STM and LTM tests in rats treated with $50 \%$ DMSO vehicle $(n=5$; black bars) or $1 \mu \mathrm{g}$ of U0126 ( $n=8$; gray bars). $\boldsymbol{b}$, Mean ( \pm SEM) retention of freezing in both groups; freezing during LTM is expressed as a percentage of that observed during the STM test. c, Mean ( \pm SEM) changes in the amplitude of LA AEFPs during the LTM test, expressed as a percentage of that obtained during the STM test. $\boldsymbol{d}$, Correlation between freezing scores and LA AEFP amplitudes in U0126-treated rats (each expressed as a percentage of STM). $\boldsymbol{e}$, Representative AEFPs in the LA for each group (vehicle, U0126) during baseline and during the LTM test. Calibration: $20 \mu \mathrm{V}, 5 \mathrm{~ms}$.

formation suggests a possible role for ERK-dependent plasticity in memory storage in the LA but is not itself conclusive. Indeed, it has been suggested that manipulations of the LA (either via lesion, inactivation, or pharmacological means) may affect fear memory formation by modulating the strength of plasticity and memory storage in regions of the wider fear network (Cahill et al., 1999). Consistent with this notion, recent studies have shown that functional inactivation of the amygdala during fear conditioning impairs the establishment of plasticity in the MGm/PIN (Maren et al., 2001; Poremba and Gabriel, 2001). Thus, it might be argued that our impairment of long-term synaptic plasticity in the LA and memory formation after intra-LA infusion of U0126 might instead reflect impaired consolidation of synaptic plasticity in the MGm/PIN, which is in turn reflected back to the LA. If true, we would expect MEK inhibition in the LA to impair longterm plastic changes in the MGm/PIN.

In our experiments, under conditions in which fear acquisition and STM were left intact, we observed no effect on learninginduced synaptic plasticity in the MGm/PIN after intra-LA infu- 


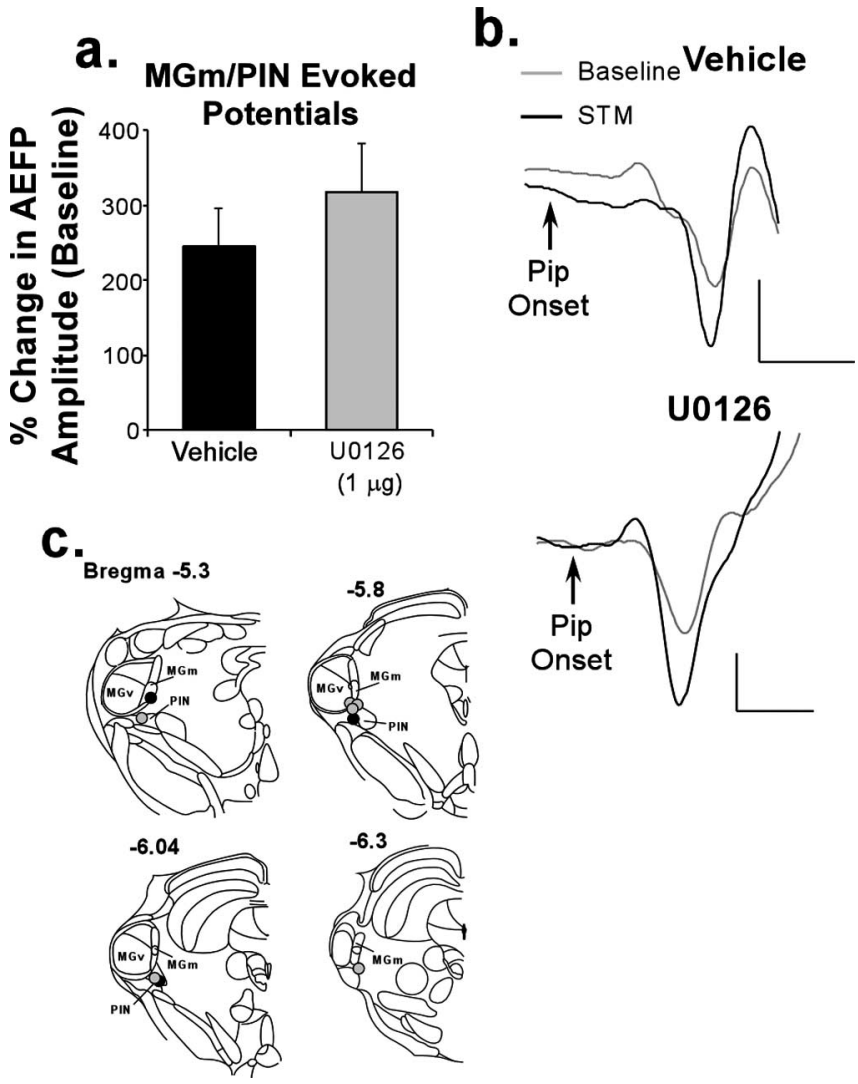

Figure 3. Intra-LA infusion of a MAPK inhibitor has no effect on short-term retention of conditioning-induced synaptic plasticity in the MGm/PIN. $\boldsymbol{a}$, Mean ( \pm SEM) percentage of change in AEFP amplitude in the MGm/PIN expressed during the STM test in rats treated with $50 \%$ DMSO vehicle ( $n=3$; black bar) or $1 \mu \mathrm{g}$ of U0126 ( $n=6$; gray bar). $\boldsymbol{b}$, Representative AEFPs in the MGm/PIN for each group (vehicle, U0126) during baseline and during the STM test. Calibration: $20 \mu \mathrm{V}, 5 \mathrm{~ms}$. c, Electrode tip placements in rats treated with vehicle (black circles) or U0126 (gray circles). MGv, Ventral division of the medial geniculate nucleus.

sion of U0126. As in the LA, auditory fear conditioning led to significant enhancement in the activity of MGm/PIN neurons relative to baseline during the STM test (vehicle: $t_{(2)}=4.58, p<$ 0.05; U0126: $\left.t_{(5)}=4.2, p<0.01\right)$ that was equivalent in both groups (Fig. $3 a)\left(t_{(8)}=0.29 ; p>0.05\right)$. Representative traces recorded from the MGm/PIN in vehicle and U0126-treated rats during the STM test are shown in Figure $3 b$, whereas electrode tip placements are shown in Figure $3 c$. These findings clearly show that intra-LA infusion of U0126 leaves the fear acquisition process intact and does not interfere with the development of conditioning-induced plasticity in either the LA or the $\mathrm{MGm} / \mathrm{PIN}$.

The remaining question is whether the neural changes observed in the LA, expressed either during STM or during LTM, are simple reflections of those induced in the MGm/PIN rather than representing plasticity induced locally at LA synapses. Our findings are inconsistent with this hypothesis. First, we observed no correlation between the change in AEFP amplitudes in the $\mathrm{MGm} / \mathrm{PIN}$ and the LA expressed during the STM test $\left(r_{(8)}=\right.$ $0.381 ; p>0.05$; data not shown). Second, if the thalamus was the source of the effects observed in the LA, then intra-LA infusion of U0126 would be also expected to impair long-term synaptic plasticity in the MGm/PIN. This effect was not observed. Of the animals from which we successfully obtained recordings from both the LA and MGm/PIN, the group differences in AEFP amplitude change recorded in the LA (Fig. $4 a)\left(t_{(7)}=3.20 ; p<0.05\right.$ )
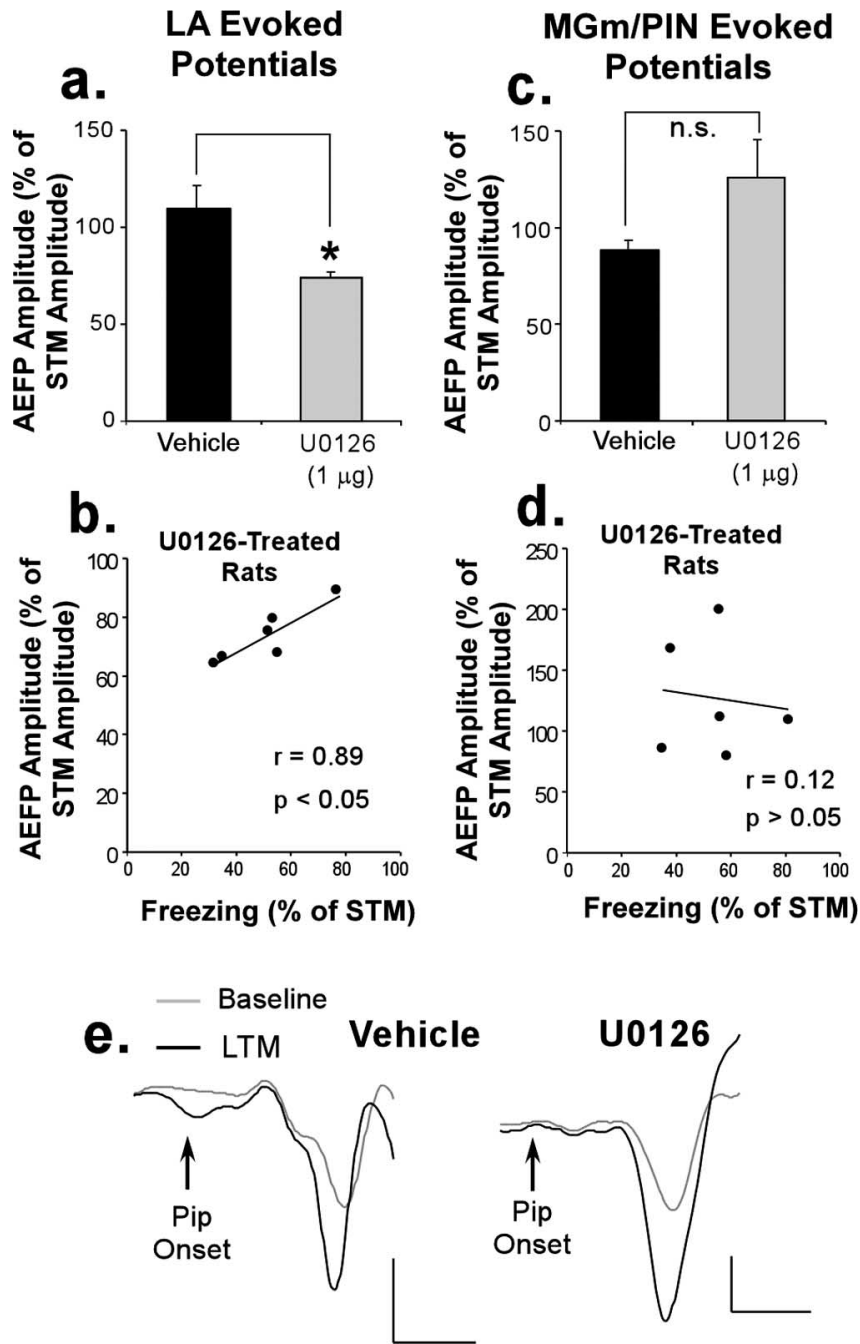

Figure 4. Intra-LA infusion of a MAPK inhibitor does not impair long-term retention of conditioning-induced synaptic plasticity in the MGm/PIN. $\boldsymbol{a}$, Mean ( \pm SEM) change in LA AEFP amplitudes during LTM (expressed as a percentage of STM) for the subset of rats treated with vehicle ( $n=3$; black bar) or U0126 ( $n=6$; gray bar) in which recordings were also made from the MGm/PIN. $\boldsymbol{b}$, Correlation between freezing and LA AEFP amplitudes of U0126-treated rats (each expressed as a percentage of STM). c, Mean ( \pm SEM) change in AEFP amplitude in the MGm/PIN during LTM (expressed as a percentage of STM) for vehicle and U0126-treated rats. $\boldsymbol{d}$, Correlation between freezing and MGm/PIN AEFP amplitudes of U0126-treated rats (each expressed as a percentage of STM). $\boldsymbol{e}$, Representative AEFPs in the MGm/PIN during baseline and the LTM test in vehicle and U0126-treated rats. Calibration: $20 \mu \mathrm{V}, 5 \mathrm{~ms}$.

and the correlation between freezing scores and percentage of change in AEFP amplitudes within the U0126-treated group remained significant $\left(r_{(5)}=0.89 ; p<0.05\right)$ (Fig. $\left.4 b\right)$. However, when the same analysis was applied to AEFPs in the MGm/PIN, no difference emerged between the two groups. Unlike the LA, the AEFP amplitudes of both groups remained similarly enhanced 24 h later $\left(t_{(7)}=1.29 ; p>0.05\right)$ (Fig. $\left.4 c\right)$. Furthermore, no significant correlation between freezing scores and percentage of change in AEFP amplitudes was found in U0126-treated rats in the MGm/PIN $\left(r_{(5)}=0.12 ; p>0.05\right)$ (Fig. $4 d$ ). Representative traces recorded at $24 \mathrm{~h}$ after training from the MGm/PIN in vehicle and U0126-treated rats are shown in Figure 4e.

These findings indicate that local inhibition of ERK/MAPK activation in the LA has no effect on the consolidation of conditioning-induced plasticity in the MGm/PIN. Thus, the impairment of long-term plasticity in the LA after infusion of U0126 
is not the result of impaired long-term plasticity in the MGm/ PIN, which is in turn reflected back to the LA.

\section{Discussion}

Previous studies have shown that fear conditioning leads to neurophysiological changes in the LA (Quirk et al., 1995; Rogan et al., 1997; Maren and Quirk, 2004). Left unresolved, however, has been the question of whether these changes reflect local synaptic plasticity in the amygdala, or whether they reflect a read-out of plasticity from other areas of the wider fear network (Cahill et al., 1999).

In the present experiments, we have examined in parallel the effects of local blockade of a key intracellular signaling cascade in the LA on both fear memory consolidation and neurophysiological changes in both the LA and MGm/PIN. The findings revealed that inhibition of ERK/MAPK in the LA selectively impairs longterm retention of plasticity at thalamo-LA synapses in correlation with the magnitude of the impairment of fear memory consolidation; it has no effect on the development and retention of thalamic plasticity. Thus, although it is clear that the LA, and possibly plasticity in the LA, is essential for the initiation of thalamic plasticity during fear conditioning (Maren et al., 2001; Poremba and Gabriel, 2001), the present findings may indicate that LA plasticity is not essential for the maintenance of thalamic plasticity once it is established.

Collectively, our findings show that long-term synaptic plasticity in the LA is required for both memory consolidation of fear conditioning and the consolidation of training-induced plasticity in the LA. Furthermore, the findings rule out the possibility that plastic changes that develop in the LA during and after fear conditioning are a mere reflection of those that occur in the MGm/ PIN (Cahill et al., 1999), suggesting that plastic changes in the $\mathrm{MGm} / \mathrm{PIN}$ alone are not sufficient for the full expression of a fear memory. These conclusions, although arrived at by different means, are consistent with those of both Poremba and Gabriel (2001) and Maren et al. (2001). In the study by Poremba and Gabriel (2001), muscimol inactivation of the amygdala impaired fear conditioning and the development of MGm/PIN plasticity. However, when the animals were retrained, fear conditioning proceeded normally without accompanying thalamic plasticity (Poremba and Gabriel, 2001). In the study by Maren et al. (2001), muscimol-treated rats with residual MGm/PIN plasticity were retrained and exhibited normal rates of reacquisition relative to controls, suggesting that residual thalamic plasticity confers no savings of fear memory. Together, these findings suggest that MGm/PIN plasticity is not essential for fear memory formation under certain circumstances.

Although our findings suggest that plasticity in the MGm/PIN may not be sufficient for fear memory formation, they should not be interpreted to suggest that the auditory thalamus plays no role in fear conditioning. Indeed, a recent study from our laboratory has shown that inhibition of ERK/MAPK or RNA synthesis, but not protein synthesis, in the MGm/PIN impairs memory consolidation of auditory fear conditioning (Apergis-Schoute et al., 2005). These findings, which are also partially consistent with those of a previous study (Maren et al., 2003), suggest that although the auditory thalamus is not a site of protein synthesisdependent memory storage per se, it still plays an important role in memory formation of auditory fear conditioning. One possibility is that the MGm/PIN contributes to fear memory formation by contributing to presynaptic aspects of memory formation in the LA, a possibility supported by the finding that ERK/MAPK inhibition in the MGm/PIN impairs LTP in the LA (ApergisSchoute et al., 2005). Additional experiments will be required for a full understanding of the role of the MGm/PIN in memory formation of fear conditioning.

In conclusion, the findings of the present study strongly suggest that long-term retention of conditioning-induced potentiation at thalamo-LA synapses is a critical requirement for the storage of a fear memory within the LA. These findings demonstrate for the first time that conditioned enhancement of CS responses in the auditory thalamus is not sufficient to support memory storage during fear conditioning, whereas conditioned enhancement of CS responses in the LA is both necessary and sufficient for memory storage. The present findings do not diminish the potential importance of the auditory thalamus and other structures in the encoding of different components of the entire fear memory trace, nor do they suggest that the amygdala plays no role in modulating certain types of memory storage. However, our findings clearly demonstrate that long-term storage of the emotional memory trace relies on local synaptic plasticity in the LA.

\section{References}

Apergis-Schoute AM, Debiec J, Doyere V, LeDoux JE, Schafe GE (2005) Auditory fear conditioning and long-term potentiation in the lateral amygdala require ERK/MAP kinase signaling in the auditory thalamus: a role for presynaptic plasticity in the fear system. J Neurosci 25:5730-5739.

Bakin JS, Weinberger NM (1990) Classical conditioning induces CSspecific receptive field plasticity in the auditory cortex of the guinea pig. Brain Res 536:271-286.

Blair HT, Schafe GE, Bauer EP, Rodrigues SM, LeDoux JE (2001) Synaptic plasticity in the lateral amygdala: a cellular hypothesis of fear conditioning. Learn Mem 8:229-242.

Cahill L, Weinberger NM, Roozendaal B, McGaugh JL (1999) Is the amygdala a locus of "conditioned fear"? Some questions and caveats. Neuron 23:227-228.

Davis M (1992) The role of the amygdala in fear-potentiated startle: implications for animal models of anxiety. Trends Pharmacol Sci 13:35-41.

Doyère V, Schafe GE, Sigurdsson T, LeDoux JE (2003) Long-term potentiation in freely moving rats reveals asymmetries in thalamic and cortical inputs to the lateral amygdala. Eur J Neurosci 17:2703-2715.

Edeline JM, Weinberger NM (1993) Receptive field plasticity in the auditory cortex during frequency discrimination training: selective retuning independent of task difficulty. Behav Neurosci 107:82-103.

Fanselow MS, LeDoux JE (1999) Why we think plasticity underlying Pavlovian fear conditioning occurs in the basolateral amygdala. Neuron 23:229-232.

Gabriel M, Saltwick SE, Miller JD (1975) Conditioning and reversal of short-latency multiple-unit responses in the rabbit medial geniculate nucleus. Science 189:1108-1109.

LeDoux JE (2000) Emotion circuits in the brain. Annu Rev Neurosci 23:155-184.

Lennartz RC, Weinberger NM (1992) Frequency-specific receptive field plasticity in the medial geniculate body induced by Pavlovian fear conditioning is expressed in the anesthetized brain. Behav Neurosci 106:484-497.

Maren S, Quirk GJ (2004) Neuronal signalling of fear memory. Nat Rev Neurosci 5:844-852.

Maren S, Yap SA, Goosens KA (2001) The amygdala is essential for the development of neuronal plasticity in the medial geniculate nucleus during auditory fear conditioning in rats. J Neurosci 21:RC135(1-6).

Maren S, Ferrario CR, Corcoran KA, Desmond TJ, Frey KA (2003) Protein synthesis in the amygdala, but not the auditory thalamus, is required for consolidation of Pavlovian fear conditioning in rats. Eur J Neurosci 18:3080-3088.

Paré D (2002) Mechanisms of Pavlovian fear conditioning: has the engram been located? Trends Neurosci 25:436-438. 
Poremba A, Gabriel M (2001) Amygdalar efferents initiate auditory thalamic discriminative training-induced neuronal activity. J Neurosci 21:270-278.

Quirk GJ, Repa C, LeDoux JE (1995) Fear conditioning enhances shortlatency auditory responses of lateral amygdala neurons: parallel recordings in the freely behaving rat. Neuron 15:1029-1039.

Quirk GJ, Armony JL, Repa JC, Li XF, LeDoux JE (1996) Emotional memory: a search for sites of plasticity. Cold Spring Harb Symp Quant Biol 61:247-257.

Rodrigues SM, Schafe GE, LeDoux JE (2004) Molecular mechanisms underlying emotional learning and memory in the lateral amygdala. Neuron 44:75-91.
Rogan MT, Staubli UV, LeDoux JE (1997) Fear conditioning induces associative long-term potentiation in the amygdala. Nature 390:604-607.

Romanski LM, LeDoux JE (1992) Bilateral destruction of neocortical and perirhinal projection targets of the acoustic thalamus does not disrupt auditory fear conditioning. Neurosci Lett, 142:228-232.

Romanski LM, Clugnet MC, Bordi F, LeDoux JE (1993) Somatosensory and auditory convergence in the lateral nucleus of the amygdala. Behav Neurosci 107:444-450.

Schafe GE, Atkins CM, Swank MW, Bauer EP, Sweatt JD, LeDoux JE (2000) Activation of ERK/MAP kinase in the amygdala is required for memory consolidation of pavlovian fear conditioning. J Neurosci 20: 8177-8187. 